\title{
Floating bare tether as upper atmosphere probe
}

\author{
J. R. Sanmartin, ${ }^{1}$ M. Charro, ${ }^{1}$ J. Pelaez, ${ }^{1}$ I. Tinao, ${ }^{1}$ S. Elaskar, ${ }^{2}$ A. Hilgers, ${ }^{3}$ \\ and M. Martinez-Sanchez ${ }^{4}$
}

[1] Use of a conductive bare tape electrically floating in low Earth orbit as an effective electron beam source to produce artificial auroral effects, free of problems that mar standard beams, is considered. Ambient ions impacting the tape with $\mathrm{keV}$ energies over most of its length liberate secondary electrons that race down the magnetic field, excite neutrals in the E layer, and result in auroral emissions. The tether would operate with both a power supply and a plasma contactor off at nighttime; power and contactor would be on at daytime for reboost. Tomographic analysis of auroral emissions from the footprint of the beam, as observed from the spacecraft, can provide density profiles of dominant neutral species in the E layer. A characteristic tether system, at altitude $\sim 300 \mathrm{~km}$ and moderate orbital inclination, would involve an aluminum tape with a length of $\sim 20 \mathrm{~km}$, a width of $\sim 15 \mathrm{~mm}$, and a thickness of $\sim 0.2 \mathrm{~mm}$ for a full-system mass around $1200 \mathrm{~kg}$, with two thirds going into the power subsystem.

\section{Introduction}

[2] Active experiments using electron beam sources on board rockets to produce artificial auroras started in 1969 [Hess et al., 1971; Davis et al., 1971]. This pioneer work was followed by a series of rocket missions such as Echo, for upward injection [Israelson and Winckler, 1975; Hallinan et al., 1978; Winckler et al., 1984], Excede, for low-altitude injection [O'Neil et al., 1978a, 1978b; Paulsen et al., 1990], and TEP/Charge, involving tethers [Sasaki et al., 1987; Myers et al., 1990; Gilchrist et al., 1990]. Beam sources have also been used onboard the shuttle [Wilhelm et al., 1984; Beghin et al., 1984; Banks et al., 1987]. The APEX experiment used two satellites at a distance that could be varied and controlled [Oraevsky and Triska, 1993]. All these experiments, using e-beams that are by now standard, could take advantage of a precise knowledge of timing/location of the beam and, in principle, of the spectrum of the precipitating electrons; lack of such knowledge had hampered natural auroral studies. Reviews of e-beam experiments were given by Winckler [1980, 1992].

[3] Standard e-beams are marred in several ways, however. Beam-firing affects the spacecraft $(\mathrm{S} / \mathrm{C})$ potential that serves as ground for the beam source. The gross perturbations produced by the intense emission in the space plasma around the spacecraft affects the beam itself, and the luminous glow arising from electron bombardment in the return current contaminates sensitive optical instruments. Beams are thin; the cross-section radius may be less than one electron gyroradius at $\mathrm{keV}$ energies, for parallel injection, thus requiring ground observation that is made possible by an energy flux up to 2 orders of magnitude greater than in the strongest natural auroras. Intense beams compensate for the thinness of the emitting layer, but they are hard to predict, produce suprathermal electrons and plasma fluctuations near the spacecraft, and are distorted by nonlinear plasma interactions that may structure the beam cross section [Cartwright et al., 1978; Sasaki et al., 1985; Banks and Raitt, 1988]. The values of beam-to-plasma density and flux ratios have been shown to be critical for nonlinear interactions [Strangeway, 1980; Okuda et al., 1987; Winglee and Pritchett, 1988]. The important case of relativistic electron beams shows strong beam-plasma interaction effects [Neubert and Gilchrist, 2004].

[4] A conductive tether left uninsulated and electrically floating in LEO might serve as an effective e-beam source [Martinez-Sanchez and Sanmartin, 1997]. Tether bias comes out negative over most of its length. Ions impacting with $\mathrm{keV}$ energies liberate secondary electrons that accelerate outward through the two-dimensional (2-D) local bias, then race down magnetic lines and result in auroral emissions in the E layer. The e-beam is free of $\mathrm{S} / \mathrm{C}$ charging problems (no current flows at the tether ends), and is free of plasma-interaction problems because both beam density and flux are very small. In addition, beam emission takes place far from any instrument.

[5] A weak flux will exhibit low brightness that makes ground observation difficult. Brightness would be greater for observation from the spacecraft, also allowing continuous measurements. This is impracticable for the thin cross 


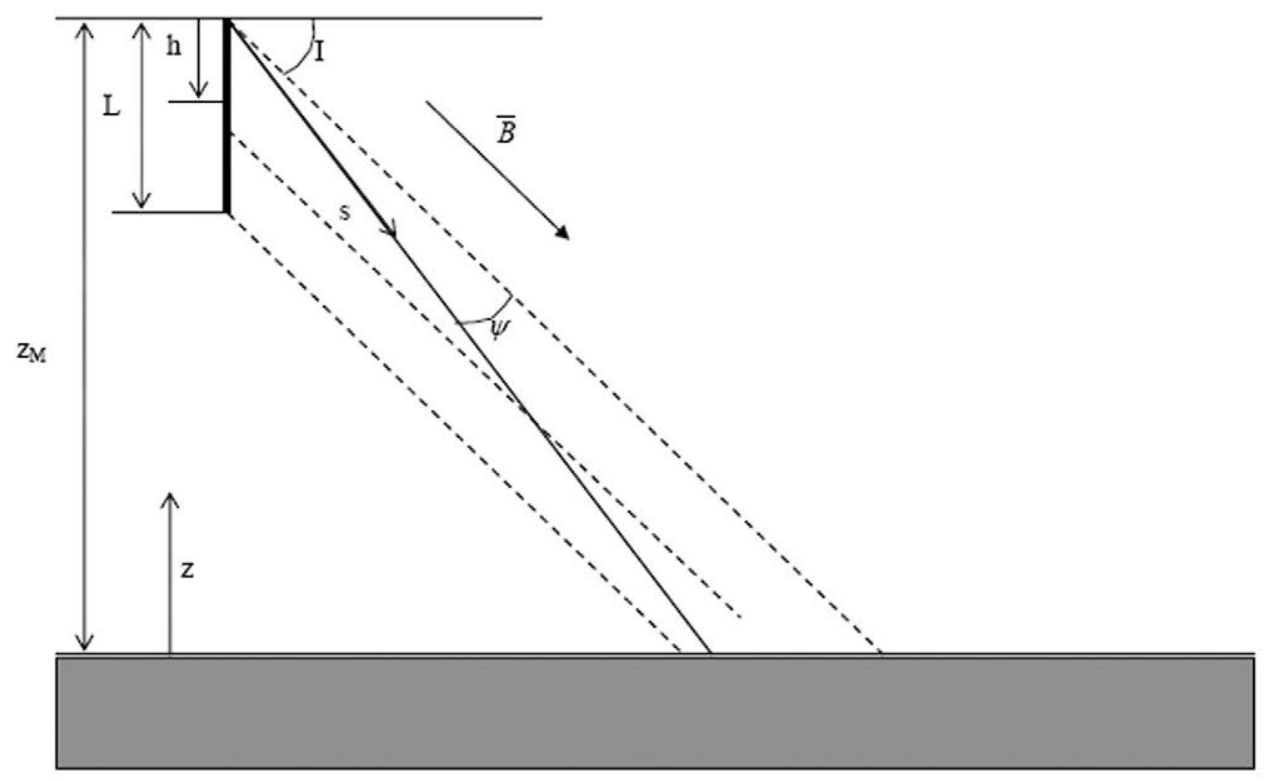

Figure 1. Schematics of emissions observation from the tether top, at a range of angle $\psi$ with respect to magnetic field lines; $I$ is the magnetic dip angle.

section of a standard beam, but the tether beam has one cross-dimension of order of tether length $L$, which is tens of kilometers (Figure 1). Each point in the tether emits monoenergetic secondary electrons, but both electron flux and energy increase near linearly with distance $h$ from top. Observation from the spacecraft along any straight line at angle $\psi$, extending some optical depth $\Delta s$ over the ionization region, mix altitude-z/h effects. As a result, the narrow footprint of the beam in the E layer might show a structure in angle $\psi$, allowing the determination of volume emission rates by tomographic techniques.

[6] There is Lorentz drag on the electron current during nighttime operation, with power supply and a hollow cathode (HC) for electrical contact with the ionosphere off. Power and $\mathrm{HC}$ at the top would be on at daytime to reverse the current along a segment of the tether and reboost the $\mathrm{S} / \mathrm{C}$, once per orbit. The supply power must produce thrust enough along that segment to balance both drag on the rest of the tether and drag during the night, resulting in an e-beam source as an autonomous system that might change its own orbit when desired. Beyond auroral effects proper, observations from the $\mathrm{S} / \mathrm{C}$ might provide real-time mapping of the neutral density in the E layer, of interest in numerical simulations of the atmosphere lying below, and in reentry calculations.

[7] Some Echo missions, in addition to the TEP/Charge missions, involved both tethers and e-beams. Tethers were also proposed in the past for high-resolution, density mapping of some ionospheric species: in a dual platform configuration, a shuttle-based laser would excite an atmosphere column adjacent to a tethered satellite, with triangulating photometric detectors on the satellite employed to measure the fluorescence from sections of the column [McComas et al., 1985]. Use of downward deployed long tethers to tow instrument packages in the E layer for in situ probing of atmospheric processes has been also discussed [Kirby, 1995]. In an e-beam proposal on sounding parallel electric-field structures within the auroral acceleration re- gion, a tether would be used to keep the beam source payload and the diagnostics away from each other, and avoid contaminating optical instruments [Habash et al., 1995].

[8] In the next section we determine the e-beam generated by a bare tether. In section 3 we discuss beam evolution and its foot-track at the E layer. In sections 4 and 5 we consider observational and tomography schemes. Tether-system design is discussed in section 6. Conclusions are presented in section 7. Some observational and dynamical issues are considered in Appendices A and B.

\section{Electron Beam at Emission}

[9] As regards observations and mass requirements, tether design is mainly concerned with values of tether length, and cross-section perimeter $p$ and area $A$. Only $L$ and $p$ values condition observations. Full bias and energy of secondary electrons are proportional to $L$; the sides of the beam cross section at emission will then scale as tether length $L$ and as gyroradius of secondaries $\sim \sqrt{ }$ energy $\sim$ $\sqrt{ } L$. As shown below, the current of secondaries $I_{\text {sec }}$ scales as $p L^{3 / 2} \times L$. Particle and energy beam-flux then scale as $p \times L$ and $p \times L^{2}$ respectively. Whatever the $L$ and $p$ values, however, the ratio $A / p$ critically affects system mass. Tether mass is reduced by using a thin tape $(p / 2 \approx$ width $w$ ) but this increases the mass of the power subsystem pushing current at daytime operation; we discuss this issue in section 6 .

[10] During operation at nighttime intervals in orbit with both $\mathrm{HC}$ and power supply off at the top A, the current vanishes at top and bottom (Figure 2). For an eastward orbit, electrons are collected above a zero-bias point $\mathrm{B}$ and ions are collected below, following the orbital-motionlimited (OML) current law in either case. Each ion picks up an electron to leave as neutral, electrons thus leaking out below $\mathrm{B}$ at the ion impact rate. At the $1-\mathrm{kV}$ bias range of interest, the leak rate increases owing to substantial secondary emission, with a yield, roughly linear with energy in the 


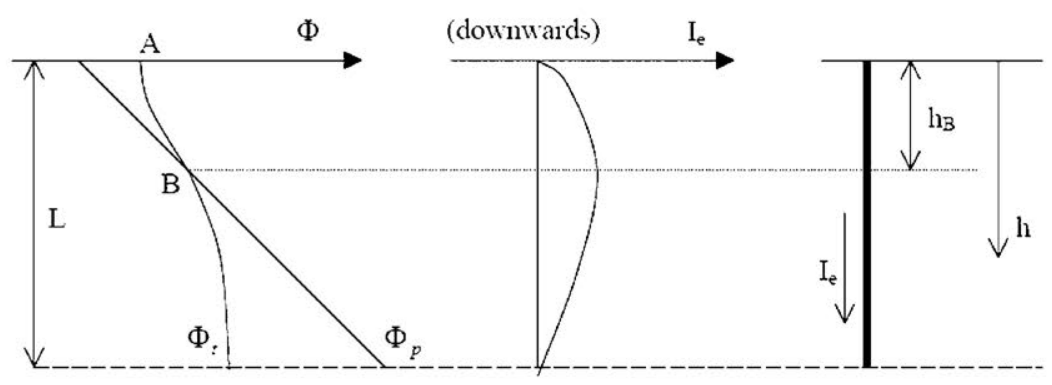

Figure 2. Night profiles of electron current, and tether and plasma potentials in the tether frame. The $\Phi_{t}$ profile has been distorted for clarity; it should be near straight and vertical, with point B very close to the top. For the parameter values in equation (4), the peak current at B is about $1.05 \mathrm{~A}$, and the peak bias at the bottom is about $3 \mathrm{kV}$.

$\mathrm{keV}$ range, of order $0.1-0.2 / \mathrm{keV}$ per impacting ion [Hastings and Garrett, 1996].

[11] The electron current $I_{e}$ in the tether flows downward everywhere, increasing from top to point $\mathrm{B}$ and decreasing from B to bottom. Bias and current profiles are determined by the equations

$$
\begin{gathered}
\frac{d \Delta V(h)}{d h}=-E_{m}+\frac{I_{e}(h)}{\sigma_{c} A}, \\
\frac{d I_{e}}{d h}=\frac{e N_{n} 2 w}{\pi} \sqrt{\frac{2 e \Delta V}{m_{e}}}, \quad\left(h<h_{B}\right) \\
\frac{d I_{e}}{d h}=-\frac{e N_{n} 2 w}{\pi} \sqrt{\frac{2 e|\Delta V|}{m_{i}}}\left[1+\gamma_{1}|\Delta V|\right], \quad\left(h>h_{B}\right)
\end{gathered}
$$

under conditions $I_{e}(0)=I_{e}(L)=0, \Delta V\left(h_{B}\right)=0$. Here $\gamma_{1}$ is yield written per unit bias; $N_{n}$ is night plasma density; and the local bias $\Delta V$ is the difference between tether potential and undisturbed plasma potential, both in the tether frame (Figure 2). A high motional field $E_{m} \equiv v_{\text {orb }} \times B_{\perp}$ (geomagnetic component perpendicular to orbital plane) requires a moderate orbital inclination $i_{\text {orb }}$. Also, for conditions in LEO, current at daytime would drop below the OML value if $w$ exceeds about $24 \mathrm{~mm}$ [Sanmartin and Estes, 1999; Estes and Sanmartin, 2000].

[12] Because the OML collection rate is proportional to the inverse square root of particle mass, which is ion mass over most of the tether, ohmic effects come out to be weak, a floating tether being near equipotential. Dropping the last term in equation (1) to set $\Delta V=E_{m}\left(h_{B}-h\right)$ allows immediate integration of equations (2a) and (2b), yielding

$$
\begin{gathered}
I_{e}\left(h_{B}\right) \approx \frac{2}{3} \frac{e N_{n} 2 w h_{B}}{\pi} \sqrt{\frac{2 e E_{m} h_{B}}{m_{e}}}, \\
\frac{h_{B}}{L} \approx\left(\frac{m_{e}}{m_{i}}\right)^{1 / 3}\left[1+\frac{3}{5} \gamma_{1} E_{m} L\right]^{2 / 3} \approx 0.039 .
\end{gathered}
$$

Here, and wherever numerical values are required, we take oxygen ions and set

$$
\begin{gathered}
\gamma_{1}=\frac{0.15}{\mathrm{kV}}, \\
E_{m}=\frac{150 \mathrm{~V}}{\mathrm{~km}}, \\
L=20 \mathrm{~km}, \\
w=16 \mathrm{~mm}, \\
N_{n}=\frac{2 \times 10^{5}}{\mathrm{~cm}^{3}} .
\end{gathered}
$$

Ohmic effects will be discussed in section 6 .

[13] We ignore the upper, positive bias segment in Figure 2 , setting $h_{B} \approx 0, I_{e}(0) \approx I_{e}\left(h_{B}\right)$ as given in equation $(3 \mathrm{a})$. We then readily find

$$
\frac{d I_{\mathrm{sec}}}{d h} \approx \gamma_{1} E_{m} h \times \frac{e N_{n} 2 w}{\pi} \sqrt{\frac{2 e E_{m} h}{m_{i}}} .
$$

We also find, for use in section 6, the drag power at night and the total secondary electron

$$
\begin{aligned}
& W_{\text {drag }}(\text { night }) \approx\left(\frac{1}{\gamma_{1}}+\frac{5}{7} E_{m} L\right) I_{\mathrm{sec}}^{\text {total }}, \\
& I_{\mathrm{sec}}^{\text {total }} \approx \frac{2}{5} \gamma_{1} E_{m} L \times \frac{e N_{n} 2 w L}{\pi} \sqrt{\frac{2 e E_{m} L}{m_{i}}} .
\end{aligned}
$$

[14] At each point $h$, secondaries are emitted with low (a few eV) energies and accelerate away from the tether under the large potential difference $|\Delta V|=E_{m} h$. At the start of the (horizontally injected) electrons race, down (or up) magnetic lines, we take the electron gyroradius $l_{b \infty}(h)=$ $\left(2 e E_{m} h / m_{e}\right)^{1 / 2} / \Omega_{e}$, which vanishes at the top of the tether, as 


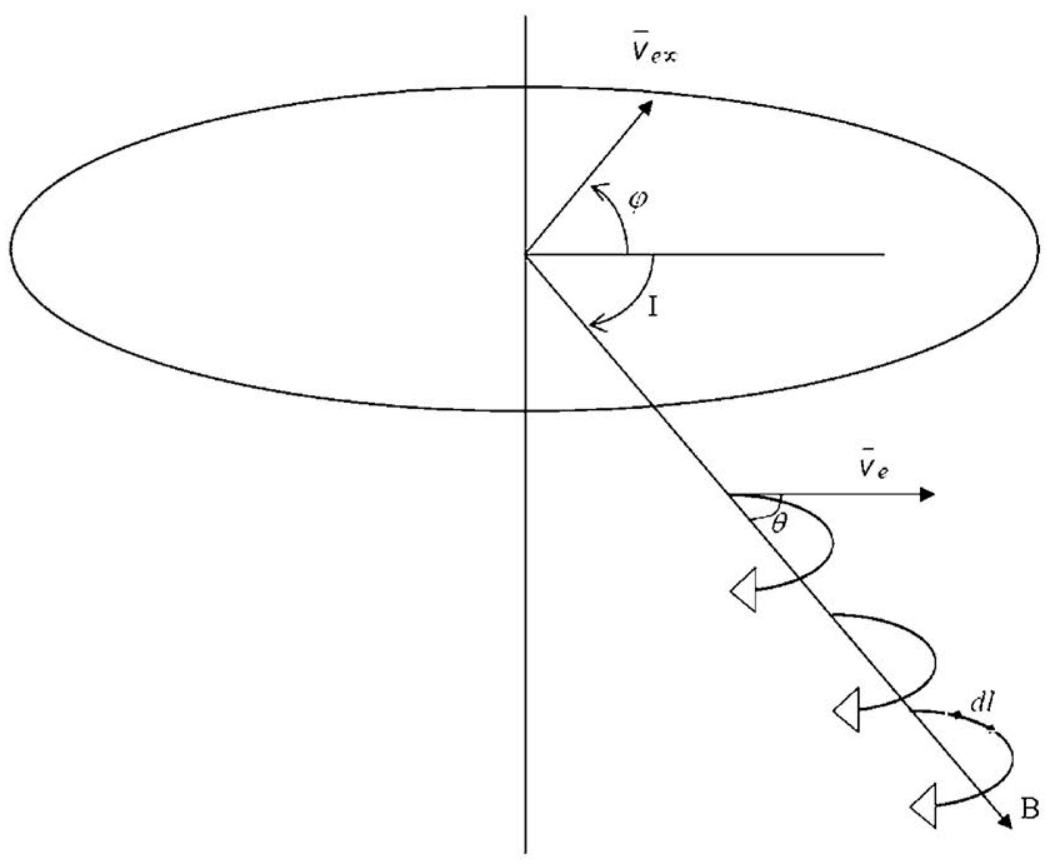

Figure 3. Geometry of secondary electron emission and pitch distribution.

beam half-width perpendicular to the vertical plane through the magnetic field $\mathbf{B}$ (Figure 3); here $\Omega_{e}$ is the gyrofrequency $e B / m_{e}$. The downward electron flux is now

$$
\Phi_{\infty}(h)=\frac{1}{2} \times \frac{d\left(I_{\mathrm{sec}} / e\right)}{2 l_{b \infty} \cos I \times d h}=N_{n} \times \Omega_{e} w \times \sqrt{\frac{m_{e}}{m_{i}}} \times \frac{\gamma_{1} E_{m} h}{2 \pi \cos I},
$$

where $I$ is magnetic dip angle. With $w$ less than the thermal gyroradius $l_{t h}=\left(k T / m_{e}\right)^{1 / 2} / \Omega_{\mathrm{e}}$, the maximum flux $\Phi_{\infty}$, at $h=L$, is smaller than the ambient electron flux by a factor much smaller than the ratio $\sqrt{ } m_{e} / m_{i}$ itself; as later shown, the flux, down at the E layer where night plasma density might be $10^{2}$ times smaller than along the orbit, is further reduced by a factor $\sim 20$ by beam broadening. Note also that there are neither sensible mirror effects nor echoes for our downward flux of secondaries.

[15] For large tether bias and OML regime conditions, the potential follows a 2-D Laplace solution for some distance away from the tape, most of the outward acceleration of secondaries thus occurring where the potential has become near radial. We then assume, as a first approximation, that emitted secondaries are uniformly distributed in azimuthal angle $\varphi$ around the tether (Figure 3). From the relation $\cos \theta=\cos I \times \cos \varphi$, one readily finds the normalized distribution in pitch angle $\theta$; using $\mu \equiv \cos \theta$, the pitchdistributed beam flux at start is

$$
\Phi_{\infty \mu}=\Phi_{\infty}(h) \times \frac{2 / \pi}{\sqrt{\mu_{I}^{2}-\mu^{2}}} \quad\left(0<\mu<\mu_{I} \equiv \cos I\right) .
$$

Writing

$$
\Phi_{\infty}(h)=\int_{0}^{\mu_{I}} N_{b} \times \sqrt{\frac{2 e E_{m} h}{m_{e}}} \mu \times \frac{(2 / \pi) d \mu}{\sqrt{\mu_{I}^{2}-\mu^{2}}}
$$

we find a very small beam-plasma density ratio,

$$
\frac{N_{b}}{N_{n}}=\sqrt{\frac{m_{e}}{m_{i}}} \times \frac{w}{l_{t h}} \frac{\gamma_{1} E_{m} h}{4 \sqrt{2} \mu_{I}^{2}} \times \sqrt{\frac{k T}{e E_{m} h}}<10^{-5} \sqrt{\frac{h}{L}} .
$$

\section{Beam Footprint at the $\mathbf{E}$ Layer}

[16] With Coulomb cross sections varying as square of energy, keV-energy secondaries are near-collisionless above $200 \mathrm{~km}$. As they move in helical paths farther down magnetic lines, they experience inelastic as well as scattering interactions with air molecules. For every ionization event there are also a number of excitation collisions followed by prompt photon emission through allowed transitions; one ionization is produced on the average for every $35 \mathrm{eV}$ of energy $\left(\varepsilon_{i}\right)$ lost by a beam electron [Rees, 1989]. Cross sections have a similar energy dependence for all interactions and are characterized by a maximum, and an energy threshold; a lumped ionization cross section can be written as

$$
\sigma_{i}(\varepsilon) \approx \sigma_{*} \times g\left(\varepsilon / \varepsilon_{*}\right)
$$

Simple modeling of cross sections [Green and Stolarski, 1972] requires $\sigma_{i}$ to vanish as $\ln \varepsilon / \varepsilon$ at high energy (Born approximation), to vanish at some threshold energy $\varepsilon_{*}$, and to have a maximum at $\varepsilon / \varepsilon_{*}$ between 4 and 5. For energy above $\varepsilon_{i}$, and for both dominant species $\mathrm{N}_{2}$ and $\mathrm{O}_{2}$, the cross section is well modeled by the shape function [MartinezSanchez and Sanmartin, 1997]

$$
\begin{aligned}
& g(u)=\frac{u-1}{u^{2}} \ln u \\
& \left(\sigma_{*} \approx 10^{-15} \mathrm{~cm}^{2}, \varepsilon_{*} \approx 23.6 \mathrm{eV} \approx 2 \varepsilon_{i} / 3\right) .
\end{aligned}
$$




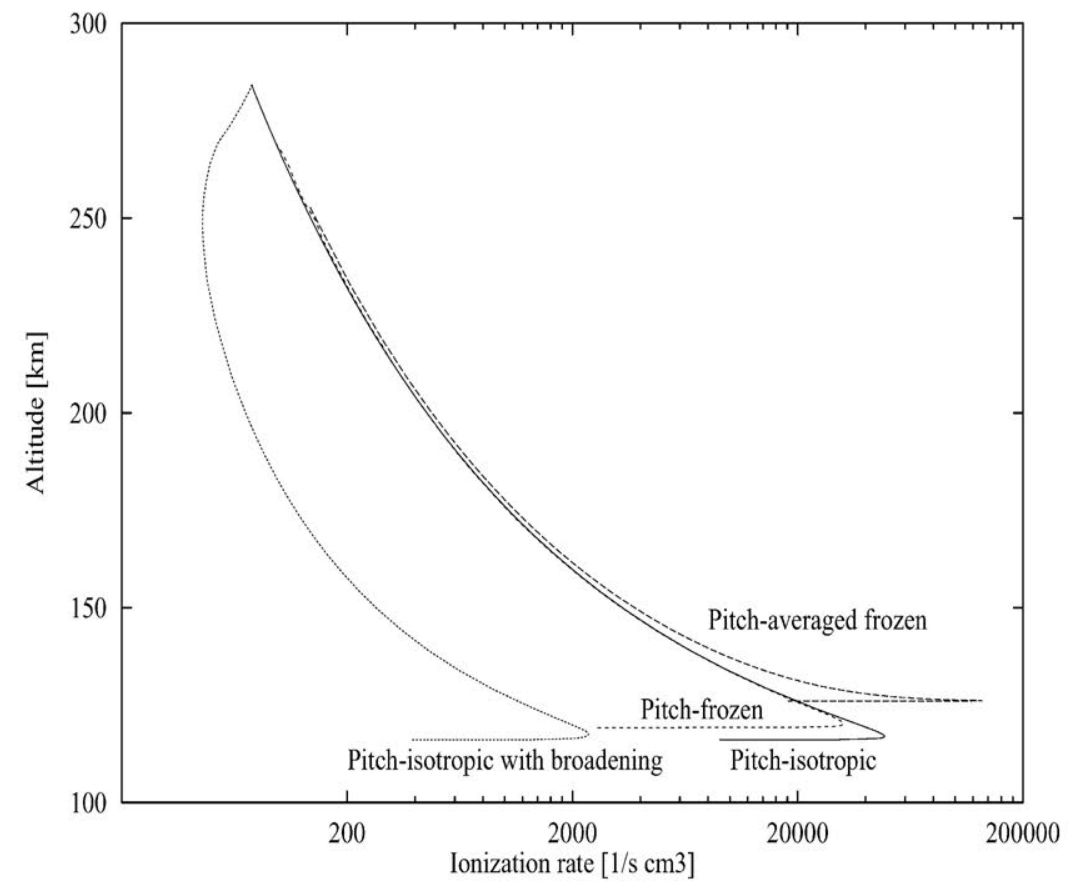

Figure 4. Altitude profiles of ionization rate for four pitch-distribution models, for $B=0.38$ gauss, $I=45^{\circ}$; parameter values given in equations (4) and (12); and neutral density profile in equation (20).

As a secondary electron with energy $\varepsilon$, pitch angle $\theta$, and mean free path $1 / n \sigma_{i}$ moves a distance $d l$ along its path, the altitude loss is $d z=-\mu \sin I d l$ and the energy loss rate is (Figure 3)

$$
\mu \sin I \frac{d \varepsilon}{d z}=\varepsilon_{i} n(z) \sigma_{i}(\varepsilon), \quad \varepsilon>\varepsilon_{i} .
$$

In a first approximation, we ignore scattering; we freeze, in particular, the pitch distribution of the beam in the initial form given by equation (8). Then equation (13) can be solved for the energy $\varepsilon(z ; h, \mu)$ at height $z$ of electrons leaving the tether at a given $h$, with the initial condition $\varepsilon\left(z_{M}-h ; h, \mu\right)=e E_{m} h$, where $z_{M}$ is altitude at the tether top (Figure 1). Writing electron energy as $\varepsilon \equiv \varepsilon_{*} \times \tilde{\varepsilon}(z ; h, \mu)$, the dimensionless function $\tilde{\varepsilon}$ is given by the equation

$$
\begin{aligned}
& \int_{\tilde{\varepsilon}}^{h / h *} \frac{d u}{g(u)}=\frac{1.5 \sigma_{*}}{\mu \sin I} \int_{z}^{z_{M}-h} n\left(z^{\prime}\right) d z^{\prime}, \\
& 1.5 \leq \tilde{\varepsilon} \leq \frac{h}{h_{*}} \quad\left(h_{*} \equiv \frac{\varepsilon_{*}}{e E_{m}}\right)
\end{aligned}
$$

with $h_{*} \approx 230 \mathrm{~m}$ for the values in equations (4b) and (12); the condition $h>1.5 h_{*} \approx 350 \mathrm{~m}$ effectively excludes nonionizing electrons from the beam.

[17] Ignoring any change in beam cross section, the volumetric ionization rate is now

$$
\dot{n}_{i}(z ; h)=\Phi_{\infty}(h) n(z) \sigma_{*} \times \int_{\mu_{\min }(z ; h)}^{\mu_{l}} \frac{(2 / \pi) d \mu}{\sqrt{\mu_{I}^{2}-\mu^{2}}} g[\tilde{\varepsilon}(z ; h, \mu)] .
$$

The lower end of the integration range, $\mu_{\min }$, is determined by setting $\tilde{\varepsilon}=1.5$ in equation (14), $\tilde{\varepsilon}\left(z ; h, \mu_{\min }\right)=1.5$.
[18] We next estimate scattering effects by assuming that electrons reach a uniform pitch-angle distribution over the range $0-\pi / 2$ immediately after leaving the tether and keep this distribution afterward. This is equivalent to setting $\mu_{I}=1$ in equation (8). Elastic collisions will also broaden the beam through diffusion perpendicular to the magnetic lines. For 1-D diffusion along the perpendicular to the vertical plane through the field $\boldsymbol{B}$ one has

$$
v_{p a r} \frac{d\left\langle R^{2}\right\rangle}{d l_{p a r}}=2 \times D_{\perp}=\frac{2}{3} \sqrt{\frac{2 \varepsilon}{m_{e}}} \frac{l^{2}}{\lambda_{s c}},
$$

where $v_{\text {par }} \equiv \mu\left(2 \varepsilon / m_{e}\right)^{1 / 2}$ is electron velocity parallel to $\boldsymbol{B}$; $d l_{\text {par }} \equiv-d z / \sin I$ is distance advanced along the field corresponding to a height decrease $d z$ (Figure 3 ); $\sqrt{ }\left\langle R^{2}\right\rangle$ is diffusion length; and $D_{\perp}$ is the diffusion coefficient perpendicular to the magnetic lines, with the mean free path $\lambda_{s c}=1 / n(z) \sigma_{s c}$ much greater than the electron gyroradius $l$.

[19] Using $l \propto \sqrt{ } \varepsilon$ and writing $l^{2}=l_{b \infty}^{2}(h) \times\left(\varepsilon / e E_{m} h\right)$, equations (13) and (16) yield

$$
\frac{d\left\langle R^{2}\right\rangle}{d \varepsilon}=-\frac{2}{3} \frac{\varepsilon}{e E_{m} h} \frac{l_{b \infty}^{2}(h)}{\varepsilon_{i} g(\tilde{\varepsilon})} \times \frac{\sigma_{s c}}{\sigma_{*}} .
$$

With $\left\langle R^{2}\right\rangle=l_{b \infty}^{2}$ at $\varepsilon=e E_{m} h$, equation (17) yields

$$
f_{b r}^{2} \equiv \frac{\left\langle R^{2}\right\rangle}{l_{b \infty}^{2}(h)}=1+\frac{4 h_{*}}{9 h} \frac{\sigma_{s c}}{\sigma_{*}} \times \int_{\tilde{\varepsilon}}^{h / h *} \frac{u d u}{g(u)} .
$$

As a result of scattering, the flux of electrons that left the tether at a given $h$ is reduced as it propagates by a factor 


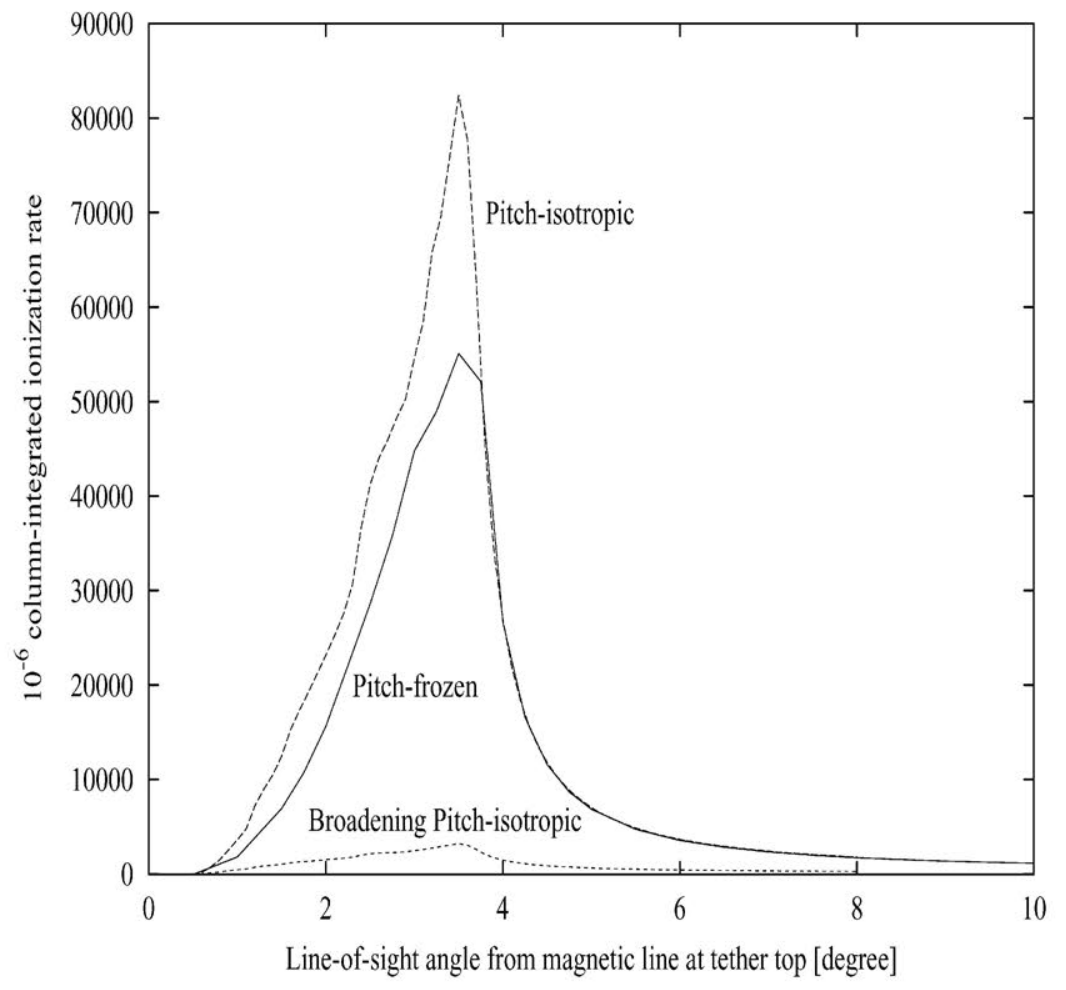

Figure 5. Column-integrated ionization rate versus observation angle, for three pitch-distribution models; parameter values are as in Figure 4.

$1 / f_{b r}$ owing to broadening. Using equation (18), the ionization rate takes now the form

$$
\dot{n}_{i}(z ; h)=\Phi_{\infty}(h) \times n(z) \sigma_{*} \times \int_{\mu_{\min }(z ; h)}^{1} \frac{(2 / \pi) d \mu}{\sqrt{1-\mu^{2}}} \frac{g[\tilde{\varepsilon}(z ; h, \mu)]}{f_{b r}[\tilde{\varepsilon}(z ; h, \mu), h]} .
$$

[20] Figure 4 compares ionization rate profiles as given by equation (15) for the pitch-frozen model and equation (19) for the pitch-isotropic/beam-broadened model. Profiles for an isotropic-pitch model that omits broadening and a frozen pitch-averaged model are also shown for illustration. We used the parameter values in equations (4) and (12), and set $I=45^{\circ}, B=0.38$ gauss, and $\sigma_{s c} \approx \sigma_{*}$ (somewhat overestimating broadening); to compute ionization we used a neutral-density profile that is reasonably valid in the altitude range 120-200 km [Carroll, 1997]

$$
n\left(\mathrm{~m}^{-3}\right)=\frac{10^{31}}{[z(m)-95,000]^{3}} .
$$

As seen in Figure 4, the model of isotropic pitch with no broadening, and the full frozen-pitch model are close to each other; broadening reduces ionization by more than an order of magnitude.

[21] For later observational considerations it is convenient to introduce the column integrated ionization rate along any straight line, $\int \dot{n}_{i} d s$, where $d s$ is the length element along the line and the integral extends over the ionization region. The lines of interest for use in the next section correspond to views from the top of the tether at a (small) angle $\psi$ with the magnetic field. Relations immediately following from Figure 1 are

$$
\begin{gathered}
\sin (I+\psi) d s=-d z \\
h=h_{\psi}(z) \equiv\left(z_{M}-z\right) \times\left[1-\frac{\tan I}{\tan (I+\psi)}\right] .
\end{gathered}
$$

We would then find, for the isotropic-pitch/beam broadened model to be used in section 5,

$$
\left.\int \dot{n}_{i} d s\right|_{\psi}=\int_{z_{\min }(\psi)}^{z_{\max }(\psi)} \frac{\dot{n}(z ; h) d z}{\sin (I+\psi)}, \quad h=h_{\psi}(z)
$$

Ionization does not occur for $z>z_{\max }(\psi)$, and $z<z_{\min }(\psi)$; these integration limits are the roots of the equation

$$
\tilde{\varepsilon}\left[z ; h_{\psi}(z), 1\right]=1.5 \rightarrow z_{\max }(\psi), z_{\min }(\psi),
$$

obtained by setting $\mu_{\min }=1$ and using (22). The two roots meet at some angle $\psi_{m}$, with $\int \dot{n}_{i} d s$ vanishing for $\psi<\psi_{m}$. Also,

$$
z_{\min }=h_{\psi}^{-1}(L), \quad \text { for } \psi>\psi_{M},
$$

where $h_{\psi}^{-1}$ is the inverse function of $h_{\psi}$, and $\psi_{M}$ is determined by the condition $\tilde{\varepsilon}\left[h_{\Psi M}^{-1}(L) ; L, 1\right]=1.5$. Actually, as shown in section 5, only values $\psi>\psi_{M}$ are used in the calculations. Figure 5 shows column-integrated 
ionization rate versus angle $\psi$ for all three pitch-distribution models, showing $\psi_{m}$ to be about 0.5 degrees. The factor $10^{-6}$ is introduced to make discussion of emission brightness in the next section easier.

\section{Observational Scheme}

[22] The beam from a bare tether has convenient features (each point in the tether ejects monoenergetic electrons, their energy increasing linearly from top to bottom), but its auroral emissions are highly localized in both space and time. In a geomagnetic no-tilt dipole model, the plane of Figure 1 would be a meridian plane, with the tether moving toward the north pole, on the right, from ascending node to point nearest the pole. The velocity perpendicular to that plane would be $v_{o r b}$ at that point and $v_{o r b} \cos i_{\text {orb }}$ at the equator. The dwell time of a beam $250 \mathrm{~m}$ thick at most, at some particular point, will be just a few tens of milliseconds throughout the orbit. This is also about the time $\mathrm{keV}$ beam electrons take to travel down from the tether.

[23] That time is much too short for beam-induced ionization to sensibly modify the plasma density. It is long enough, however, for the population of neutrals in excited states with lifetimes $\sim 10^{-7} \mathrm{~s}$ (due to prompt emission through allowed transitions) to reach steady conditions. Such transitions include the prominent $N_{2}^{+}$first negative spectral bands, at $427.8 \mathrm{~nm}$ and $391.4 \mathrm{~nm}$, and the 777.4 and $844.6 \mathrm{~nm}$ lines and bands from atomic and molecular oxygen. The case for forbidden transitions (relevant examples being the $557.7 \mathrm{~nm}$ green line, and the $630.0 / 636.4 \mathrm{~nm}$ red doublet, of atomic oxygen) is different; we will ignore any such emissions. The ratio of auroral intensity at $630.0 \mathrm{~nm}$ to intensity at $391.4 \mathrm{~nm}$ has been used in the past to determine the characteristic energy of the incident beam electrons, but that energy is known a priori in our case.

[24] At steady state, emission rates will be determined by, and proportional to, excitation rates. With cross sections for ionization and excitations about just differing in crosssection maximum, and in energy at maximum and at threshold, simple approximate relations between emission and ionization rates have been established for prominent spectral bands and lines in natural auroras. In particular, about 70-75 ion-electron pairs are produced for every $N_{2}^{+}$-band, $\lambda=427.8 \mathrm{~nm}$ photon emitted, and 25 pairs in the case of the $\lambda=391.4 \mathrm{~nm}$ band [Carlson and Egeland, 1995]. Writing $\dot{n}_{e m}(\lambda) \approx c_{\lambda} \times \dot{n}_{i}$ with a different constant $c_{\lambda}$ for each line or band, we shall simply use results on ionization rate $\dot{n}_{i}(z ; h)$ to determine 'surface brightness' as measured in Rayleigh units, which is proportional to column-integrated emission rate,

$$
b_{\lambda}(R) \equiv 10^{-6} \times \int \dot{n}_{e m}(\lambda) d s=c_{\lambda} \times 10^{-6} \int \dot{n}_{i} d s
$$

with rate and depth of (optically thin) emission in cgs units. Brightness of $1 R$ corresponds to $4 \pi \times 10^{-6}$ photons per $\mathrm{cm}^{2}$, per stereoradian, per second, reaching a detector.

[25] Ground observation across the propagating beam would be impracticable because the energy flux is weak and the beam thin. If broadening is ignored, the optical depth is the beam thickness, $\Delta s \sim 10 \mathrm{~m}$, and $\dot{n}_{i}(\max ) \approx$
$50,000 / \mathrm{cm}^{3} \mathrm{~s}$ (Figure 4); for a typical value $c \sim 1 / 50$, equation (26) then yields brightness $b \sim 1 R$. Broadening increases $\Delta s$ by a factor $20-25$ but decreases $\dot{n}_{i}$ (max) by a similar factor. There are sources of light in the night sky from other particle precipitation into the atmosphere, even at low and middle latitudes, making for background noise that could mask any $b \sim 1 R$ effect.

[26] An auroral layer would clearly appear most intense from the ground when viewed at a high elevation angle. Observation from the $\mathrm{S} / \mathrm{C}$ would provide line-of-sights along the beam equivalent to maximum elevation angle. One may then directly use our results of section 3 to determine "surface brightness" at each angle $\psi$ from the magnetic field; using column-integrated ionization rates for the broadened beam (Figure 5) and $c \sim 1 / 50$, equation (26) yields $b \sim 30-100 R$. For use in section 5 , we explicitly rewrite (normalized) brightness at angle $\psi$ as

$$
\begin{aligned}
\bar{b}_{\lambda}(\psi) & \equiv \frac{\sin (I+\psi) \tan (I+\psi)}{\tan (I+\psi)-\tan I} \frac{10^{6} b_{\lambda \psi}(R)}{c_{\lambda} \Phi_{\infty}(L)} \\
& =\int_{z_{\min }(\psi)}^{z_{\max }(\psi)} n(z) \sigma_{*} d z \times \frac{z_{M}-z}{L} \\
& \times \int_{\mu_{\min }[z ; h]}^{1} \frac{(2 / \pi) d \mu}{\sqrt{1-\mu^{2}}} \frac{g[\tilde{\varepsilon}(z ; h, \mu)]}{f_{b r}[\tilde{\varepsilon}(z ; h, \mu), h]}, \quad h=h_{\psi}(z) .
\end{aligned}
$$

[27] The $\mathrm{S} / \mathrm{C}$ altitude $z_{M}$ is restricted by the needs to reduce air drag and to locate the tether in the $F 1$ ionospheric layer (to attain maximum $e$-beam intensity); in the present calculations, we set $z_{M}=305 \mathrm{~km}$, with the $20 \mathrm{~km}$ tether reaching down to $285 \mathrm{~km}$ altitude, on the basis of preliminary safety considerations regarding the International Space Station. As regards orbital inclination, moderate $i_{\text {orb }}$ values are required, as noted in section 2 . In the geomagnetic no-tilt dipole model one has $0.5<\tan I<2$ over nearly 80 per cent of an $i_{\text {orb }}=45^{\circ}$ orbit. Figure 6 compares values for column-integrated ionization rates at fixed $E_{m}$ and values $\tan I=0.5,1$, and 2 , showing a moderate dependence on dip angle. As suggested in the figure, the footprint will reach further in angle- $\psi$ at $I \approx 45^{\circ}$; from Figure 1 we have

$$
\frac{\left(z_{M}-z_{e m}\right)-L}{z_{M}-z_{e m}} \approx \frac{\tan I}{\tan \left(I+\psi_{f p}\right)},
$$

where $\psi_{f p}$ is the angle subtending the footprint at some characteristic altitude of emission $z_{e m}$; for $L \ll z_{M}-z_{e m}$ we then find $\psi_{f p}$ maximum at $\tan I \approx 1$.

[28] In a first simple scheme one would register luminosity at three wavelengths in order to determine all three dominant densities, $N_{2}$ (at $\lambda=391.4 \mathrm{~nm}$, or $427.8 \mathrm{~nm}$ bands), and $O$ and $O_{2}$ (at $\lambda=777.4 \mathrm{~nm}$ and $844.6 \mathrm{~nm}$, with definite branching ratios). One might use three $\mathrm{CCD}$-based cameras, each with a single filter, or a single camera with hardware-controlled adaptive filters that can switch rapidly from one wavelength to the other. Note, however, that the image of the beam footprint will be very narrow; it may extend tens of kilometers horizontally in the vertical plane of Figure 1, whereas it is only $200-250 \mathrm{~m}$ horizontally across. As an alternative, a camera with a grating could map 


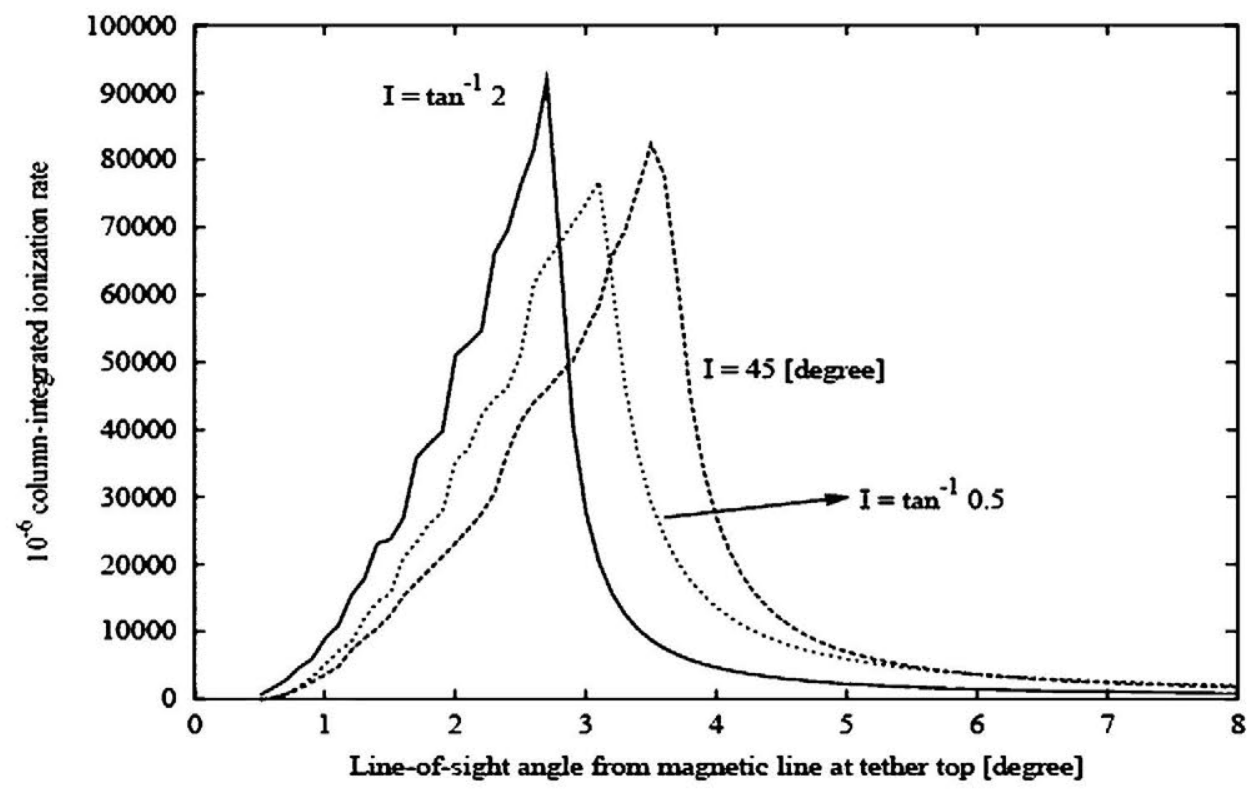

Figure 6. Column-integrated ionization rate for the pitch-isotropic model; parameter values are as in Figure 4, with two additional values of magnetic dip angle $I$.

the narrow images of the different wavelengths into different areas of the detector [Paxton and Meng, 1999]; in this way, one dimension of the array would provide spectral information while the other dimension provides spatial information, in the so-called multidimensional imaging.

[29] Resolution of the $n(z)$ profile will require a large number $N_{\text {pix }}$ of pixels in each side of the CCD-array, about $10^{3}$ in a standard $10 \mathrm{~mm} \times 10 \mathrm{~mm}$ chip, with pixel size $l_{p i x}$ $\approx 10 \mu \mathrm{m}$. As noted below, the signal-to-noise ratio increases as $l_{\text {pix }}^{2}$. For $l_{\text {pix }}=30 \mu \mathrm{m}$, the detector side length would be

$$
l_{\text {det }}=N_{\text {pix }} \times l_{\text {pix }} \sim 10^{3} \times 30 \mu \mathrm{m} \approx 3 \mathrm{~cm} .
$$

One could tile nine CCD chips to get a detector of size $3 \mathrm{~cm} \times 3 \mathrm{~cm}$, with the signal read in parallel and recombined by software. The focal length $f$ is now determined by the angular field of view, $\theta_{f v}=l_{\text {det }} / f$. Figure 5 shows most emission falling within a $6^{\circ}$ angle; to ease alignment requirements, we consider a field of view twice as large $\left(\theta_{f v} \approx 0.2\right)$, leading to

$$
f \approx l_{\text {det }} / \theta_{f v} \approx 15 \mathrm{~cm}
$$

[30] Both exposure time $\tau_{\exp }$ and $f$-ratio (between focal length and entrance aperture $D$ ) are also critical factors in determining the number of photons reaching a pixel,

$$
N_{\text {ph }}(\text { pixel })=\frac{10^{6}}{4 \pi} \times b(R) \times l_{\text {pix }}^{2}\left(\mathrm{~cm}^{2}\right) \times \Omega(\text { sr }) \tau_{\exp }(s) T_{\text {optics }},
$$

where $T_{\text {optics }}$ is the transmission of the system optics, as affected by losses due to reflections and filter attenuation, and $\Omega \approx \pi /(2 f / D)^{2}$ is the solid angle subtended at the detector by the entrance aperture. Standard fast cameras reach as low as 1.4 without unacceptable image distortion; here we just write $f / D \sim 1$. Note that both the Rayleigh criterion on angular resolution,

$$
\theta_{p i x} \equiv \frac{l_{p i x}}{f}>\theta(\text { Rayleigh }) \approx \frac{\lambda}{D} \Rightarrow \frac{l_{p i x}}{\lambda} \approx 20>\frac{f}{D},
$$

and the condition for negligible depth-of-field $\Delta s(\sim s)$ offfocusing,

$$
\frac{\Delta s}{s} \times \frac{f D}{s}<l_{p i x}, \Rightarrow s>\frac{f D}{l_{p i x}} \approx 1 \mathrm{~km},
$$

are well satisfied

[31] The exposure time cannot be too long because of the $\mathrm{S} / \mathrm{C}$ motion. We will here tentatively consider $\tau_{\exp } \sim 1 \mathrm{~s}$, corresponding to a satellite displacement of $7.5 \mathrm{~km}$, atmospheric emission being reasonably homogeneous over such horizontal distances. With $T_{\text {optics }} \sim 0.5$, equation (30) yields

$$
N_{p h}(\text { pixel }) \approx 0.3 b(R) .
$$

For $b=30-100 R$, one then gets $N_{p h}($ pixel $) \sim 10-30$. With present values of CCD quantum efficiency, $\eta$ (number of electrons per photon) $\approx 1$, the charge packet of a pixel would contain typically 20 electrons.

[32] Recall now that the image of the beam footprint, about $200 \mathrm{~m}$ wide across, will be very narrow, this horizontal resolution being unnecessary for the purposes of measurements. The view angle across, $200 \mathrm{~m} / 200 \mathrm{~km} \approx$ 0.001 , is 5 times as large as $\theta_{p i x}$ in equation (31). One might combine several pixels into a super pixel, summing the photons gathered by nearby pixels across the length of the image, in a binning mode with no increase in readout noise. The number of electrons in a super pixel would be

$$
\eta N_{\text {ph }}(\text { spixel }) \sim 100 .
$$


[33] Background noise is well below brightness values $b$ $\sim 30-100 R$. Also, noise due to dark current (pixel electrons escaping spontaneously under their own thermal energy) should be negligible; at a temperature $-20^{\circ}, \mathrm{CCD}$ dark current might be about 0.1 electrons per pixel for a 1-s exposure. Regarding CCD readout, the generated photoelectrons are collected at the output register and converted to an electrical signal (a voltage that is amplified, further digitized, and recorded as a number of counts or data numbers) with a readout (rms) error on number of electrons $R$. The signal-to-noise ratio is then

$$
S / N=\frac{\eta N_{p h}(\text { spix })}{\sqrt{\eta N_{p h}(\text { spix })+R^{2}}} .
$$

Subelectron readouts, $R^{2}<1$, can be attained at present. Equation (35) then yields $S / N \approx 10$, which may be too low. A larger $S / N$ ratio might be achieved by increasing, say, brightness $b$ in equation (30) through use of a longer tether, or by introducing an image intensifier, which performs a preamplification of the signal with a large net gain.

\section{Tomographic Scheme}

[34] A range of tomographic inversion algorithms dealing with atmospheric emissions have been worked out. They depend heavily on the observation system used. A basic problem in optical tomography is a deficiency in information, resulting from limitations in the number of lines of sight, the angular range, and the level of noise in projections [Alpatov and Romanovsky, 1998]. This makes it necessary, in general, to include a priori information in the reconstruction algorithms, such as assuming that $n(z)$ is smooth [Craig and Brown, 1986].

[35] Auroral Large Imaging System (ALIS) is a Swedish ground system using six automated stations to locate auroral phenomena by triangulation; the inversion algorithm yields volumetric emission rates using an iterative reconstruction analysis that considers measurements from the entire set of stations [Aso et al., 2000]. The Explorer satellites performed limb scans using a Cormack inversion [Cormack, 1963] that involved sets of observation-line integrals from different satellite locations [Solomon et al., 1984]. Limb measurements are less contaminated by backscatter effects of diffuse light coming from clouds or snow on the Earth surface than nadir scans.

[36] Our measurements are nadir scans. The problem of how to remove backscatter effects in nadir satellite photometry has been addressed in the past [Hays and Anger, 1978]. Volumetric scatter in the atmosphere has been taken into account using observations that are not fully nadir, which result in an apparent displacement of the emission layer, that is, a parallax effect [Abreu and Hays, 1981]; limb and nadir observations have been combined to determine the Earth albedo [Solomon et al., 1985]. In our case the horizontal extent of the emission layer is small (a thin ribbon about $20 \mathrm{~km}$ wide and $200 \mathrm{~m}$ thick), and backscatter may be ignored.

[37] To focus on the problem of inversion, we now consider a single species and omit subscript $\lambda$ in equation (27). Taking as unknowns a set of $N_{p i x}$ density values at selected heights, $\hat{n}_{r} \equiv n\left(z_{r}\right), r=1, \ldots, N_{\text {pix }}$, numerical-quadrature rules with proper interpolation lead from equation (27) to a vector equation,

$$
\bar{b}_{s} \equiv \bar{b}\left(\psi_{s}\right)=\sum_{r=1}^{N_{p i x}} \hat{K}_{s r}^{j} \hat{n}_{r}^{j+1}, \quad s=1, \ldots, N_{p i x}
$$

written in an iterative scheme, with $j$ the iteration step, $\psi_{s}$ the line-of-sight angle for the $s^{\text {th }}$ pixel, and $\hat{K}$ a kernel matrix evaluated using the density at step $j$. This kernel is found to be numerically singular, with linear combinations of rows or columns as small as the round-off error in the machine carrying out the calculation. Although $\hat{K}$ can be regularized [Herman et al., 1987], the iteration process does not converge unless the initial guess for the density profile lies unrealistically close to the actual profile, due apparently to the highly nonlinear dependence of $\hat{K}$ on the density profile.

[38] These difficulties can be bypassed by taking into account the geometry of the problem from start. We introduce unknown densities for definite altitude layers,

$$
n(z) \equiv n_{s} \quad \text { for } \quad z \in\left(z_{s-1}, z_{s}\right] \quad\left(z_{0} \equiv z_{M}\right)
$$

where $z_{s} \equiv h_{\psi / s}^{-1}(L)$, as shown in Figure 7 . The resulting equation is similar to equation (36), but the matrix $K$ now involved is lower triangular, making forward substitution possible,

$$
\begin{gathered}
\bar{b}_{s}=\sum_{r=1}^{N_{p i x}} K_{s r}^{j} n_{r}^{j+1}, \quad s=1, \ldots, N_{p i x}, \\
K_{s r}^{j}=K_{s r}^{j}\left(\psi_{s} ; n_{1}^{j}, \ldots, n_{r}^{j}\right), \quad r \leq s ; \quad K_{s r}^{j}=0, \quad r>s .
\end{gathered}
$$

Instead of iterating the whole set we can now iterate each row $s$ until $n_{s}$ converges, using updated density values as soon as they become available, as in a Gauss-Seidel iteration.

[39] For $s=1$, equation (38) reads just

$$
\bar{b}_{1}=K_{11}^{j}\left(\psi_{1} ; n_{1}^{j}\right) \times n_{1}^{j+1}
$$

where

$$
\begin{aligned}
K_{s 1}^{j}\left(\psi_{s} ; n_{1}^{j}\right) \equiv & \int_{z_{1}}^{z_{\max s 1}^{j}} \sigma_{*} d z \times \frac{z_{M}-z}{L} \times \\
& \int_{\mu_{\min s 1}^{j}}^{1} \frac{(2 / \pi) d \mu}{\sqrt{1-\mu^{2}}} \times \frac{g\left(\tilde{\varepsilon}_{s 1}^{j}\right)}{f_{b r}\left[\tilde{\varepsilon}_{s 1}^{j}, h_{\psi s}(z)\right]},
\end{aligned}
$$

with $\tilde{\varepsilon}_{s 1}^{j}\left(n_{1}^{j}, z ; \psi_{s}, \mu\right)$ obtained from

$$
\int_{\tilde{\varepsilon}_{s 1}^{j}}^{h_{\psi s s}(z) / h *} \frac{d u}{g(u)}=\frac{1.5 \sigma_{*}}{\mu \sin I} \frac{\tan I}{\tan \left(I+\psi_{s}\right)}\left(z_{M}-z\right) n_{1}^{j},
$$

and $\tilde{\varepsilon}_{11}^{j}\left(n_{1}^{j}, z ; \psi_{1}, \mu_{\min 11}^{j}\right)=1.5, \tilde{\varepsilon}_{11}^{j}\left(n_{1}^{j}, z_{\max 11}^{j} ; \psi_{1}, 1\right)=1.5$. Starting with a seed density $n_{1}^{0}=0$, iteration converges to some value $n_{1}^{f}$. 


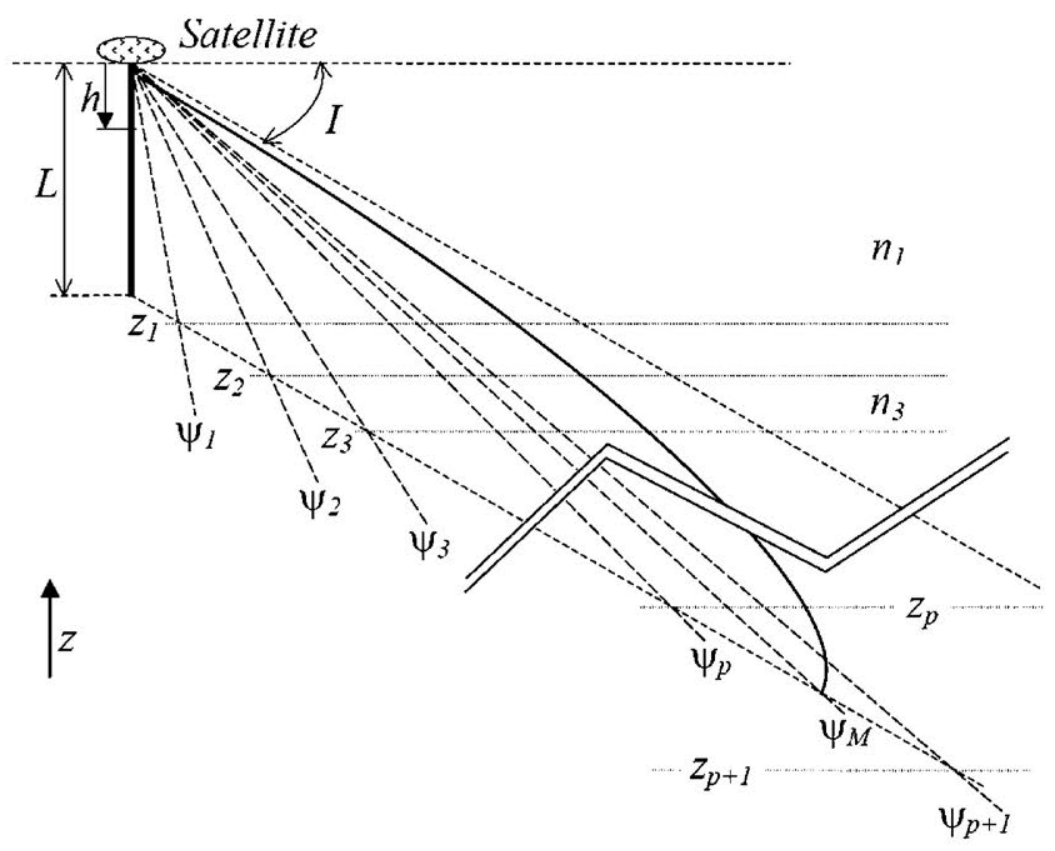

Figure 7. Schematics of neutral density and line-of-sight modeling for tomographic inversion; beyond the full line, electron energy is too small for ionization.

[40] For $s=2$, equation (38) gives

$$
\bar{b}_{2}-K_{21}^{f}\left(\psi_{2} ; n_{1}^{f}\right) \times n_{1}^{f}=K_{22}^{j}\left(\psi_{2} ; n_{1}^{f}, n_{2}^{j}\right) \times n_{2}^{j+1}
$$

with

$$
\begin{aligned}
K_{22}^{j}\left(\psi_{2} ; n_{1}^{f}, n_{2}^{j}\right)= & \int_{z_{2}}^{z_{1}} \sigma_{*} d z \times \frac{z_{M}-z}{L} \times \int_{\mu_{\text {min } 21}^{j}}^{1} \frac{(2 / \pi) d z}{\sqrt{1-\mu^{2}}} \\
& \times \frac{g\left[\tilde{\varepsilon}_{22}^{j}\right]}{f_{b r}\left[\tilde{\varepsilon}_{22}^{j}, h_{\psi 2}(z)\right]},
\end{aligned}
$$

with $\tilde{\varepsilon}_{22}^{j}\left(n_{2}^{j}, z ; \psi_{2}, \mu_{\min 22}^{j}\right)=1.5$ and $\tilde{\varepsilon}_{22}^{j}$ given by

$$
\int_{\tilde{\varepsilon}_{22}^{j}}^{\tilde{\varepsilon}_{21}^{f}\left(z_{1}\right)} \frac{d u}{g(u)}=\frac{1.5 \sigma *}{\mu \sin I}\left(z_{1}-z\right) n_{2}^{j},
$$

where $\tilde{\varepsilon}_{21}^{f}\left(z_{1}\right)$ is obtained by setting $s=2, z=z_{1}$, and $n_{1}^{j}=n_{1}^{f}$ in equation (41). Iteration proceeds from an initial seed density $n_{2}^{0}=n_{1}^{f}$.

[41] One may proceed forward determining $n_{3}^{f}, n_{4}^{f}, \ldots$ until a line of sight $s=p$ as shown in Figure 7 is reached. The full line curve in the figure represents equation (24). Each line of sight cuts it twice, as advanced in section 5, except for $\psi<\psi_{m}$, which is the angle corresponding to the tangent to the curve from the top of the tether. The line of sight reaching the curve at the magnetic line through the tether bottom corresponds to the angle $\psi_{M}$, here lying somewhere between the $p$ and $p+1$ lines. Forward determination stops here, since all lines (or pixels) following $p$ do not involve lower densities.
[42] Values of brightness for such lines might, in principle, be used to check consistency in the set of values $n_{1}^{f}$, $n_{2}^{f}, \ldots n_{p-1}^{f}$, and start a new cycle of calculations. We have found, however, that the present method works quite well at its simplest; typically $\psi_{M}$ corresponds to half way up the brightness peak (see Figure 5), the method thus using a number of pixels more than $3 / 4 N_{p i x}$. As an example, we computed brightness for the density profile given in (20), and then applied the above method to get back the density profile by interpolation in the set $n_{1}^{f}, n_{2}^{f}, \ldots n_{p-1}^{f}$ obtained. We considered two values, $N_{p i x}=100$ and 2,000; otherwise we took values of parameters as used throughout our analysis, in particular $L=20 \mathrm{~km}$ and $I=45$ degrees. Figure 8 gives the relative error, showing that it is less than $1 \%$ in the altitude range of interest except very close to $120 \mathrm{~km}$. The error decreases as the algorithm progresses down, finally bouncing back where density gradients are too large for our approximating the density as piecewise-constant; the error can be reduced by increasing $N_{p i x}$. A small value of the ratio $\varepsilon_{i} n_{1} \sigma_{*} \times\left(z_{M}-z_{1}\right) / e E_{m} L$ helps in reducing effects of the crude approximation $n \equiv n_{1}$ for $z_{1}<z<z_{M}$ at lower altitudes.

[43] Note that the sequence of altitudes $z_{s}$ in (37) follows from the sequence of angles $\psi_{s}$, which is fully determined by optics and CCD array, and that the number of equations used in (38) is less than $N_{p i x}$. In the first scheme (equation (36)), however, $N_{p i x}$ equations are used, with all $N_{\text {pix }}$ altitudes selected so as to operate on some line of sight. Apparently, this gives rise to the singularity found in the $\hat{K}$ kernel.

\section{Tether Design}

[44] As noticed in section 2, there might exist an optimal tape thickness $\delta$ that minimizes the system mass. At large enough $\delta$, hardware mass, which accounts for end-ballast/ 


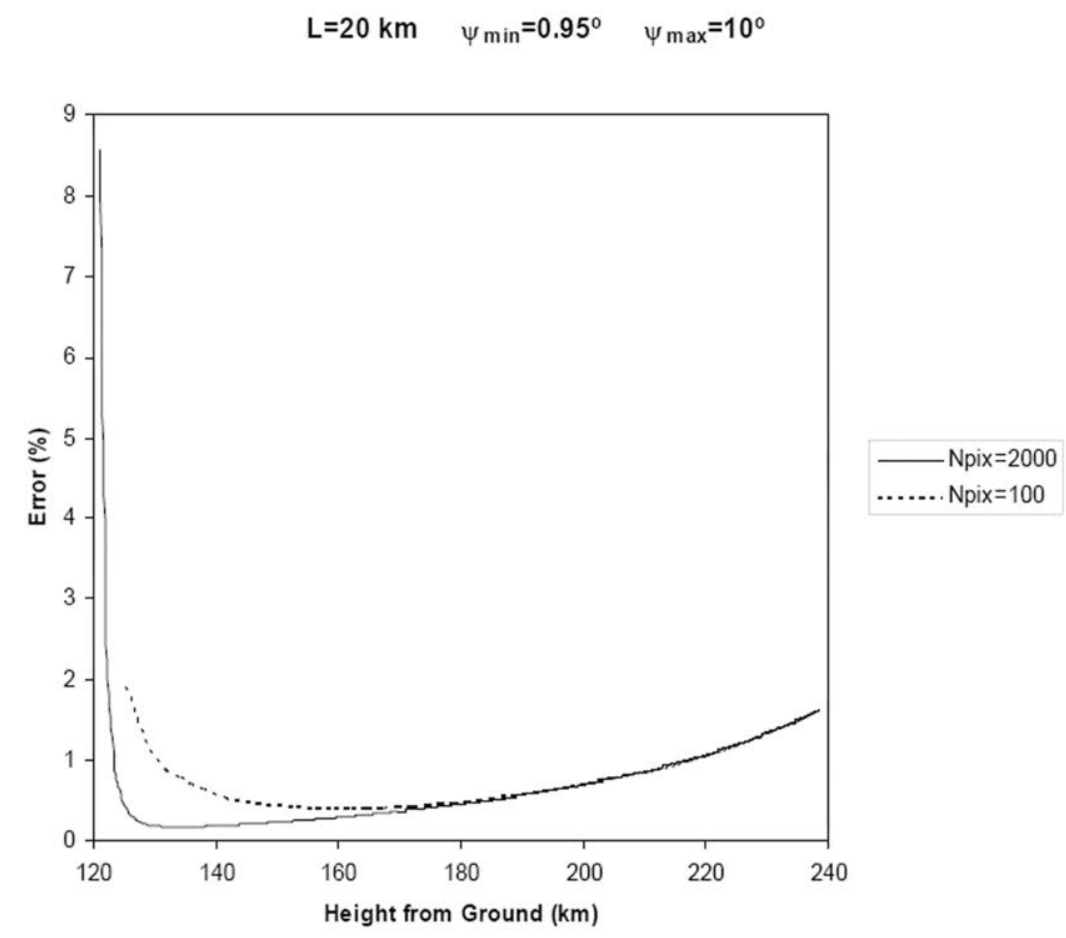

Figure 8. Relative error in the tomographic determination of density profile; parameter values are as in Figure 4.

deployer and tether itself, is clearly dominant. At small enough $\delta$, the mass of the power-subsystem (HC/Power Processing Unit/Solar Array) would be dominant. A balance of overall drag and thrust work over a full orbit yields a simple estimate of the optimal thickness.

[45] At daytime, power supply and $\mathrm{HC}$ are on to revert the current in some upper segment CA (Figure 9). The electron current flows and increases upward above $\mathrm{A}$; it flows downward below $\mathrm{A}$, increasing from $\mathrm{A}$ to $\mathrm{B}$ and decreasing from $\mathrm{B}$ to tether bottom. The condition for day thrust applied above A to balance day drag applied below $\mathrm{A}$, and night drag in Figure 2, is then

$$
(1-r) W_{\text {drag }}(\text { night })+r W_{\text {drag }}(\text { day })=r W_{\text {thrust }}(\text { day })
$$

where $W_{\text {drag }}($ day $), W_{\text {thrust }}($ day $)$ are drag and thrust power at day, and $r \approx 0.6$ is day-time fraction in orbit. We ignore here the tendency for the orbit to become increasingly elliptical, as resulting from night-drag, and net day-thrust, roughly acting on opposite orbital arcs.

[46] A calculation of day drag is simple if ohmic effects are negligible below $A$. Conditions below the zero-current point $\mathrm{A}$ in Figure 9 are then as in Figure 2, with a scaling $L \rightarrow l$ and a consideration of changes in orbital conditions, which are basically an increase in plasma density by an order of magnitude. One then finds from equations (5) and (6)

$$
\frac{W_{\text {drag }}(\text { day })}{W_{\text {drag }}(\text { night })} \approx \frac{N_{d}}{N_{n}}\left(\frac{l}{L}\right)^{5 / 2} \frac{1+\frac{5}{7} \gamma_{1} E_{m} l}{1+\frac{5}{7} \gamma_{1} E_{m} L} .
$$

[47] To determine thrust above A, where conductive effects prove definitely important, we consider equations

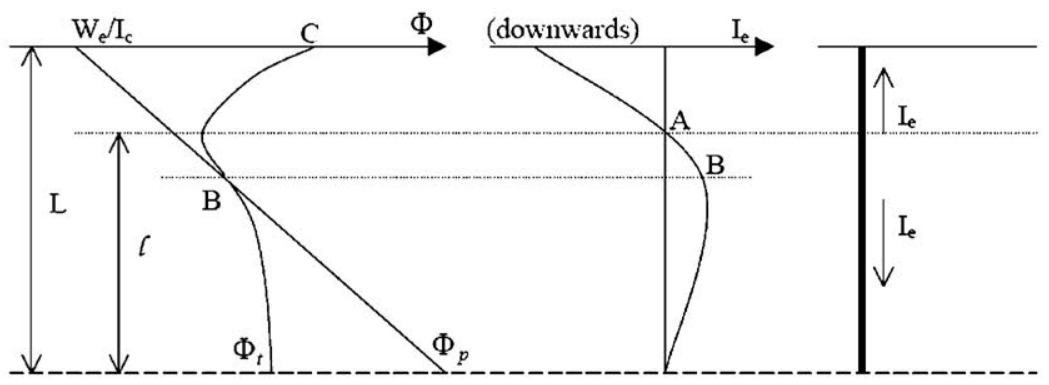

Figure 9. Daytime profiles of current, and tether and plasma potentials in tether frame. For a day density of $10^{6} \mathrm{~cm}^{-3}$, a tape thickness of $\approx 0.19 \mathrm{~mm}$, and the parameter values in equation (4), current and bias at the tether top are $46.0 \mathrm{~A}$ and $0.88 \mathrm{kV}$, respectively. 
(1) and (2a), where now $I_{e}$ is negative, and define dimensionless variables standard in bare-tether analysis,

$$
\begin{gathered}
i \equiv \frac{-I_{e}}{\sigma_{c} E_{m} A}, \\
\phi \equiv \frac{\Delta V}{E_{m} L^{*}}, \\
\xi \equiv \frac{h}{L^{*}},
\end{gathered}
$$

where $\sigma_{c}$ is tether conductivity and $L^{*}$ is a characteristic length that gauges ohmic impedance against bare-tether collection impedance and is defined for daytime density by

$$
\frac{e N_{d} 2 w L_{d}^{*}}{\pi} \sqrt{\frac{2 e E_{m} L_{d}^{*}}{m_{e}}} \equiv \frac{3}{4} \sigma_{c} E_{m} A
$$

[Sanmartin et al., 1993]. The ratio $L / L^{*} \equiv \tilde{L}$ is a fundamental dimensionless parameter for current and bias profiles along an electrodynamic bare tether.

[48] Ignoring the segment $\mathrm{AB}$ to use conditions $i=0$, $\phi \approx 0$ at $L_{d}^{*} \times \xi_{A}=L-l$, equations (1) and (2a) give

$$
\begin{gathered}
-d \phi / d \xi=1+i, . \\
2 i+i^{2}=\phi^{3 / 2}
\end{gathered}
$$

Equation (49b) immediately yields the required supply power in terms of the dimensionless bias at the top $\phi_{C}$

$$
W_{e}=\left|I_{e C}\right| \Delta V_{C}=\sigma_{c} E_{m}^{2} A L_{d}^{*} \times \phi_{C}\left(\sqrt{1+\phi_{C}^{3 / 2}}-1\right) .
$$

Also, equation (49b) may be used to integrate equation (49a) from $\mathrm{A}$ to $\mathrm{C}$ giving

$$
\xi_{A} \equiv \tilde{L}_{d}\left(1-\frac{l}{L}\right)=\int_{0}^{\phi_{C}} \frac{d \phi}{\sqrt{1+\phi^{3 / 2}}}
$$

Finally, the thrusting power can be readily calculated as

$$
\frac{W_{\text {thrust }}(\text { day })}{\sigma E_{m}^{2} A L_{d}^{*}}=\phi_{C}-\int_{0}^{\phi_{C}} \frac{d \phi}{\sqrt{1+\phi^{3 / 2}}} ;
$$

using equations (46) and (52) in equation (45) yields

$$
\begin{aligned}
\frac{3}{10} \sqrt{\frac{m_{e}}{m_{i}}} \times & \left(1+\frac{5}{7} \gamma_{1} E_{m} L\right) \\
& \times\left[\frac{1-r}{r} \frac{N_{n}}{N_{d}}+\left(\frac{l}{L}\right)^{5 / 2} \frac{1+\frac{5}{7} \gamma_{1} E_{m} l}{1+\frac{5}{7} \gamma_{1} E_{m} L}\right] \tilde{L}_{d}^{5 / 2} \\
& =\phi_{C}-\int_{0}^{\phi_{C}} \frac{d \phi}{\sqrt{1+\phi^{3 / 2}}}
\end{aligned}
$$

Equations (51) and (53) determine the thrust length-ratio $l / L$ and the dimensionless voltage at the power source, $\Delta V_{C}$ $E_{m} L_{d}^{*}$, as functions of the dimensionless tether length at day, $\tilde{L}_{d} \equiv L / L_{d}^{*}$, for given values of ion mass, time-weighted night-to-day plasma density ratio $(1-r) N_{n} / r N_{d}$, and secondary yield at full bias $\gamma_{1} E_{m} L$. We find that $\phi_{C}$ is a rapidly increasing function of $L / L_{d}^{*}$ whereas $l / L$ is nearly constant $(\approx 0.85)$ over a broad $L / L_{d}^{*}$ range.

[49] System mass can now be written as made of tether hardware and power subsystem masses, $M_{s y s} \approx \alpha_{t} \rho A L+$ $\alpha W_{e}$, where the dimensionless coefficient $\alpha_{t}=2-3$ takes into account tether end-ballast/deployer mass and $\alpha$ is inverse specific power. We are neglecting the mass of expellant, which is consumed at a $\mathrm{HC}$ at an extremely low rate. Using equation (50) we can rewrite $M_{s y s}$ as

$$
\sqrt{\frac{m_{e}}{m_{i}}} \frac{\gamma_{1}}{\alpha} \frac{3 N_{n}}{10 N_{d}} \times \frac{M_{s y s}}{I_{\text {sec }}^{\text {total }}}=\frac{\alpha_{t} \rho / \alpha \sigma E_{m}^{2}}{\tilde{L}_{d}^{3 / 2}}+\phi_{C} \frac{\sqrt{1+\phi_{C}^{3 / 2}}-1}{\tilde{L}_{d}^{5 / 2}} .
$$

With $\rho / \sigma E_{m}^{2} \approx 3.75 \mathrm{~kg} / \mathrm{kW}$ for $A l$, we have a ratio $\alpha_{t} \rho / \alpha \sigma_{c} E_{m}^{2} \approx 0.47$ for $\alpha_{t}=2.5$, and $\alpha$ as low as $5 \mathrm{~kg} / \mathrm{kW}$ for $\mathrm{HC}$ and PPU plus $15 \mathrm{~kg} / \mathrm{kW}$ for the solar array.

[50] For given emitted current $I_{\text {sec }}^{\text {total }}$, system mass is a function of $L_{d}$, with $\phi_{C}\left(\tilde{L}_{d}\right)$ determined as above. The righthand side of equation (54) exhibits a minimum that is very flat, allowing for broad design choices. Also, the minimum typically occurs at high $\tilde{L}_{d} \sim 20$, at higher $\hat{L}_{d}$ the higher is $\alpha_{t} \rho_{c} / \alpha \sigma E_{m}^{2}$ and the lower is $\gamma_{1} E_{m} L$ or $(1-r) N_{n} / r N_{d}$. Around the minimum, the mass of the power subsystem is very close to two thirds of total, $\varphi_{C}$ is about 5.9 , and the thrust efficiency is $\eta \equiv W_{\text {thust }}($ day $) / W_{e} \approx 0.17$.

[51] For $N_{d}=10^{6} \mathrm{~cm}^{-3}$, equation (48) gives $L_{d}^{*}(\mathrm{~km}) \approx$ $(0.188 \mathrm{~mm} / \delta)^{2 / 3}$, our $20 \mathrm{~km}$ long tape corresponding to $\tilde{L}_{d} \approx$ 20 for thickness $\delta \approx 0.188 \mathrm{~mm}$. The $16 \mathrm{~mm}$ wide tape would then have a mass $m_{t} \approx 162 \mathrm{~kg}$; power subsystem mass, total system mass, and electric power, would be $\alpha W_{e}$ $\approx 810 \mathrm{~kg}, M_{s y s} \approx 1215 \mathrm{~kg}$, and $W_{e} \approx 40.5 \mathrm{~kW}$, respectively. Notice that minimum system mass scales as $I_{\text {sec }} \sim L^{5 / 2}$, increasing very fast with increasing tether length; the range of design values for $L$ is narrow $(15-25 \mathrm{~km})$ because the beam energy flux $\left(\sim L^{2}\right)$ decreases rapidly with decreasing tether length. Note that the beam will reach further down in the $\mathrm{E}$ layer the longer the tether.

[52] Ohmic effects below $A$ are small, as we assumed in section 2 , if the maximum current, at point $B$, is small compared with the short-circuit current. We find

$$
\left.\frac{I_{e}}{\sigma_{c} E_{m} A}\right|_{B} \approx \frac{1}{2}\left(\frac{l}{L} \tilde{L}_{d}\right)^{3 / 2} \sqrt{\frac{m_{e}}{m_{i}}} \approx 0.2
$$

which is reasonably small. Ohmic effects at night can be shown to be weaker by one order of magnitude.

\section{Conclusions}

[53] We have shown that a thin aluminum tape of length $\sim 20 \mathrm{~km}$, width $\sim 15 \mathrm{~mm}$, and thickness $\sim 0.2 \mathrm{~mm}$, left electrically floating at both ends at night, at altitude $300 \mathrm{~km}$ and moderate orbital inclination, could serve as an effective electron beam source to produce artificial auroral 
effects. Tomographic analysis of auroral emissions from the footprint of the beam, as observed from the spacecraft, could provide density profiles of dominant neutral species in the E layer. Using a solar array and a hollow cathode on at day, to revert the current and reboost the $\mathrm{S} / \mathrm{C}$ once per orbit, would result in an autonomous system capable of very long missions. The full-system mass would be around $1200 \mathrm{~kg}$ with two thirds going into the power subsystem.

[54] Careful calibration of tether yield and measurements of plasma density at night would be required. Three-axis inorbit measurements of the geomagnetic field would be also required, in order to determine the values of dip angle, component perpendicular to the orbital plane, and electron gyrofrequency. It might be worth carrying out detailed analyses of tradeoffs between using a round wire, properly scaled up in length and down in perimeter, instead of our optimal tape, and using an ion thruster, instead of the tether itself, to produce day thrust in case of short auroral missions.

\section{Appendix A}

[55] The signal-to-noise ratio for brightness in side-view observation could be increased by time-modulating the tether current and bias, with detection conveniently phaselocked. This would require pulsing a second $\mathrm{HC}$ located at the tether bottom, reducing the bias there to a very low value. With this $\mathrm{HC}$ on, however, electron collection would take place over a substantial tether segment, increasing night drag and thus the power-subsystem mass; furthermore, this second $\mathrm{HC}$ and its subsystems would be located away from the power supply at the top.

[56] Independently, and aside from transients related to the $\mathrm{HC}$ on/off operation, transients in the bias/current pulses travelling along the tether could affect the response of ambient ions, which determine the workings of the tether as a secondary electron beam source. In studying such pulses, the tether can be modeled as a transmission line. The equations describing the time-dependent bias and current profiles are simple extensions of equations (1) and (2):

$$
\begin{gathered}
\frac{\partial \Delta V}{\partial h}=-E_{m}+R_{l} I_{e}+L_{l} \frac{\partial I_{e}}{\partial t}, \\
\frac{\partial I_{e}}{\partial h}=G_{l} \Delta V+C_{l} \frac{\partial \Delta V}{\partial t} .
\end{gathered}
$$

Here $R_{l}$ is $1 / \sigma_{\mathrm{c}} A$ and $G_{l}$ is the conductance per unit tether length of the transmission line, as given by the right-hand side of equations (2a) and (2b) if steady conditions have been reached; note that $G_{l}$, being bias-dependent, varies along the line and will be time-dependent during transients.

[57] The capacitance per unit length would be $C_{l}=2 \pi \varepsilon_{0} /$ $\ln \left(r_{s h} / R_{e q}\right)$, which is weakly dependent on the argument of the logarithm, where $r_{s h}$ is a characteristic "sheath" radius related to the Debye length $\lambda_{D}$, and $R_{e q}$ is some equivalent radius. Under steady, OML current conditions, one has $R_{e q}=w / 4$ for a tape; the argument of the logarithm is of the order of $\sqrt{ } e|\Delta V| / k T \gg 1$. Similarly, the inductance per unit length is $L_{l}=\ln \left(r_{m} / R_{e q}\right) / 2 \pi \varepsilon_{0} \mathrm{c}^{2}$, with the distance $r_{m}$ in the logarithm somewhat larger and dependent in currentcircuit closure in the ambient plasma.
[58] Ignoring the conductance term and logarithmic changes in both $C_{l}$ and $L_{l}$, the classical transmission-line equation is recovered,

$$
\frac{\partial^{2} I_{e}}{\partial h^{2}}=C_{l} L_{l} \frac{\partial^{2} I_{e}}{\partial t^{2}}+C_{l} R_{l} \frac{\partial I_{e}}{\partial t} .
$$

The "no-loss" phase velocity of the line is

$$
v_{p h}=\frac{1}{\sqrt{C_{l} L_{l}}}=c \times \sqrt{\frac{\ln \left(r_{s h} / R_{e q}\right)}{\ln \left(r_{m} / R_{e q}\right)}} \approx c,
$$

and the time for waves to travel halfway of an $L=20 \mathrm{~km}$ line, $t_{w}=L / 2 c \approx 3 \times 10^{-5} \mathrm{~s}$, is comparable to the time for $\mathrm{O}^{+}$ions to respond to changes in bias, which is a few times $r_{s h} / \sqrt{2 e|\Delta V| / m_{i}} \approx 2 \lambda_{D} / \sqrt{2 k T_{e} / m_{i}} \sim 10^{-5}$ s for $N_{n} \sim 10^{5} \mathrm{~cm}^{-3}$ [Sanmartin and Estes, 1999]. Further, for such times the ratio between the last two terms in equation (A2) is the ratio between the line resistance $R_{l} L \approx 190 \Omega$, and the ("no-loss") impedance of the line,

$$
\begin{aligned}
\sqrt{L_{l} / C_{l}}= & \sqrt{\ln \left(r_{s h} / R_{e q}\right) \times \ln \left(r_{m} / R_{e q}\right)} / 2 \pi \varepsilon_{0} c \approx 60 \Omega \\
& \times \log \text { arithmic factor },
\end{aligned}
$$

which are comparable. Pulses should therefore be sensibly attenuated.

\section{Appendix B}

[59] In general, the Lorentz torque makes a conductive tether carrying a current unstable in its motion relative to the center of mass. This skip-rope instability, involving in-plane and out-of-plane dynamics, is very sensible to current intensity. We now show that such effects are completely negligible in our case. In the simplest analysis, current is kept constant and the geomagnetic field is a no-tilt dipole, with both Lorentz and gravity-gradient forces varying as the inverse cube of orbital radius. Tether attitude then exhibits an unstable periodic motion with the period of the orbit, the largest positive eigenvalue of the monodromy matrix being [Pelaez et al., 2000]

$$
\lambda_{m} \approx 1+\frac{\pi}{9} \cos i_{o r b} \sin ^{2} i_{o r b} \varepsilon^{3} .
$$

Here

$$
\begin{gathered}
\varepsilon \equiv \frac{B_{e q} \int_{0}^{L} I_{e}(h)(h-\mu L) d h / L}{\left[\mu(1-\mu) M_{S / C}-m_{t} / 6\right] g_{0} L / R_{E}}, \\
\mu=\frac{m_{b o t}+m_{t} / 2}{M_{S / C}},
\end{gathered}
$$

where $g_{0}$ and $B_{e q}$ are acceleration of gravity and equatorial magnetic field at the Earth surface, $R_{E}$ is the Earth radius, and we are using a dumbbell model of masses, with the full spacecraft mass $M_{S / C}$ made of tether, bottom $\left(m_{b o t}\right)$, and top $\left(>\alpha W_{e}\right)$ masses. 
[60] At night, with the current profile $I_{e}(h)$ as discussed in section 2 (Figure 2 ), $\varepsilon$ would vanish in case $\mu \approx 5 / 14$, but it is very small whatever the mass distribution. For the daytime current profile (Figure 9) $\varepsilon$ vanishes at no $\mu$-value (day drag is almost as large as thrust, which acts on a short tether segment), but it decreases with distance $\mu L$ from center of mass to top. Minimum $\mu$ corresponds to just endballast/deployer bottom mass, $m_{\text {bot }}=\left(\alpha_{t}-1\right) m_{t}$, yielding $\mu=2 m_{t} / M_{S / C}$.

[61] The denominator of equation (B2a) is now

$$
2 m_{t} g_{0}(1-\mu-1 / 12) L / R_{E} \approx 7.5 \mathrm{~N},
$$

where we used values from section 6 and set $\mu=1 / 6$ $\left(M_{S / C}=1.6 M_{\text {sys }} \approx 2000 \mathrm{~kg}\right.$ ), the result, however, varying little with $\mu$ if small. Note that $L / 6$ is close to $L-l \approx 0.15 l$ in Figure 9. Then, using $B_{e q} \approx 1.5 B_{\perp}$ and $6 W_{\text {drag }}($ day $) \approx$ $5 W_{\text {thrust }}($ day), with thrust acting at about $0.05 \mathrm{~L}$ from top, the numerator of equation $(\mathrm{B} 2 \mathrm{a})$ becomes

$$
\begin{gathered}
1.5\left[\frac{5}{14} \frac{l}{L} \frac{W_{\text {drag }}(\text { day })}{v_{\text {orb }}}+0.1 \frac{W_{\text {thrust }}(\text { day })}{v_{\text {orb }}}\right] \\
\approx 1.5 \times 0.35 \times \frac{\eta W_{e}}{v_{\text {orb }}} \approx 0.5 \mathrm{~N},
\end{gathered}
$$

leading to $\varepsilon \sim 0.07$, or $\lambda_{m}-1 \sim 4 \times 10^{-5}$ for $i_{\text {orb }}=$ 45 degrees.

[62] Acknowledgments. This work was supported by the European Space Agency, under ESTEC contract 17384/03/. We are grateful to both reviewers for helpful comments.

[63] Amitava Bhattacharjee thanks Brian Gilchrist and Susumi Sasaki for their assistance in evaluating this paper.

\section{References}

Abreu, V. J., and P. B. Hays (1981), Parallax and atmospheric scattering effects on the inversion of satellite auroral observation, Appl.Opt., 20, 2203.

Alpatov, V. V., and Y. A. Romanovsky (1998), Methods of optical tomography for remote sensing of the atmosphere and near-Earth space, $A d v$. Space Res., 21, 1437.

Aso, T., A. Steen, U. Brändström, B. Gustavsson, A. Urashima, and M. Ejiri (2000), ALIS: A state-of-the-art optical observation network for the exploration of polar atmospheric processes, Adv. Space Res., 26 , 917.

Banks, P. M., and W. J. Raitt (1988), Observations of electron beam structure in space experiments, J. Geophys. Res., 93, 5811.

Banks, P. M., W. J. Raitt, A. B. White, R. I. Bush, and P. R. Williamson (1987), Results from the vehicle charging and potential experiments on STS-3, J. Spacecr: Rockets, 24, 138.

Beghin, C. J., J. P. Lebreton, B. N. Maehlum, P. I. J. Troim, and J. L. Michau (1984), Phenomena induced by charged particle beams, Science, $225,188$.

Carlson, H. C., and A. Egeland (1995), The aurora and the auroral ionosphere, in Introduction to Space Physics, edited by M. G. Kivelson and C. T. Russell, pp. 459-502, Cambridge Univ. Press, New York.

Carroll, J. A. (1997), Guidebook for analysis of tether applications, in Tethers in Space Handbook, 3rd ed., edited by M. L. Cosmo, and E. C. Lorenzini, NASA Contract Rep. NASA-CR-97-206807, 152-186.

Cartwright, D. G., S. J. Monson, and P. J. Kellogg (1978), Heating of the ambient ionosphere by an artificially injected electron beam, J. Geophys. Res., 83, 16.

Cormack, A. M. (1963), Representation of a function by its line integrals with some radiological applications, J. Appl. Phys., 34, 2722.

Craig, I. J. D., and J. C. Brown (1986), Inverse Problems in Astronomy, Adam Hilger, Bristol, U. K.

Davis, T. N., T. J. Hallinan, G. D. Mead, J. M. Mead, M. C. Trichel, and W. N. Hess (1971), Artificial aurora experiment: Ground-based optical observations, J. Geophys. Res., 76, 6082.
Estes, R. D., and J. R. Sanmartin (2000), Cylindrical Langmuir probes beyond the orbital-motion-limited regime, Phys. Plasmas, 7, 4320.

Gilchrist, B. E., P. M. Banks, T. Neubert, P. R. Williamson, N. B. Myers, W. J. Raitt, and S. Sasaki (1990), Electron collection enhancements arising from neutral gas jets on a charged vehicle in the ionosphere, J. Geophys. Res., 95, 2469.

Green, A. E. S., and R. S. Stolarski (1972), Analytic models of electron impact excitation cross sections, J. Atmos. Terr: Phys., 34, 1703.

Habash, L. A., T. Neubert, B. E. Gilchrist, and W. J. Raitt (1995), The use of electron beam experiments on orbiting tethered platforms for remote sensing of $\mathrm{e}_{\|}$in the auroral acceleration region: The Active Magnetospheric Particle Acceleration Satellite (AMPAS), paper presented at 4th International Conference on Tethers in Space, NASA, Washington, D. C., 10-14 April.

Hallinan, T. J., H. C. Stenbaek-Nielsen, and J. R. Winckler (1978), The Echo 4 Electron Beam Experiment: Television observation of artificial auroral streaks indicating strong beam interactions in the high-latitude magnetosphere, J. Geophys. Res., 83, 3263.

Hastings, D., and H. Garrett (1996), Spacecraft Environment Interactions, Cambridge Univ. Press, New York.

Hays, P. B., and C. D. Anger (1978), Influence of ground scattering on satellite auroral observations, Appl. Opt., 17, 1898

Herman, G. T., H. K. Tuy, K. J. Langenberg, and P. Sabatier (1987), Basic Methods for Tomography and Inversion Problems, Adam Hilger, Bristol, U. K.

Hess, W. N., M. C. Trichel, T. N. Davis, W. C. Beggs, G. E. Kraft, E. Stassinopoulos, and E. J. R. Maier (1971), Artificial aurora experiment: Experiment and principal results, J. Geophys. Res., 76, 6067.

Israelson, G. A., and J. R. Winckler (1975), Light production and electron scattering from electron beams artificially injected into the ionosphere, J. Geophys. Res., 80, 3709.

Kirby, K. P. (1995), Atmospheric science with tethers: Heating, cooling and chemistry in the lower thermosphere, paper presented at 4th International Conference on Tethers in Space, NASA, Washington, D. C., 10-14 April.

Martinez-Sanchez, M., and J. R. Sanmartin (1997), Artificial auroral effects from a bare conducting tether, J. Geophys. Res., 102, 27,257.

McComas, D. J., H. E. Spence, R. R. Karl, H. G. Horak, and T. D. Wilkerson (1985), Bistatic LIDAR experiment proposed for the shuttle/tethered satellite system missions, Rev. Sci. Instrum., 56, 670.

Myers, N. B., W. J. Raitt, A. B. White, P. M. Banks, B. E. Gilchrist, and S. Sasaki (1990), Vehicle charging effects during electron beam emission from the CHARGE-2 experiment, J. Spacecr: Rockets, 27, 25.

Neubert, T., and B. E. Gilchrist (2004), Relativistic electron beam injection from spacecraft: Performance and applications, Adv. Space Res., 34 , 2409.

Okuda, H., R. Horton, M. Ono, and M. Ashour-Abdalla (1987), Propagation of a nonrelativistic electron beam in a plasma in a magnetic field, Phys Fluids, 30, 200

O'Neil, R. R., F. Bien, D. Burt, J. A. Sandock, and J. A. T. Stair (1978a), Summarized results of the artificial auroral experiment, PRECEDE, $J$. Geophys. Res., 83, 3273.

O'Neil, R. R., O. Sheperd, W. P. Reidy, J. W. Carpenter, T. N. Davis, D. Newell, J. C. Ulwick, and J. A. T. Stair (1978b), EXCEDE II test, an artificial auroral experiment: Ground-based optical measurements, J. Geophys. Res., 83, 3281 .

Oraevsky, V. N., and P. Triska (1993), Active plasma experiment: Project APEX, Adv. Space Res., 13, 103.

Paulsen, D. E., A. J. Ratkowski, F. Bien, L. C. Howlett, I. L. Thurgood, I. L. Kofsky, W. Boquist, and R. E. Murphy (1990), EXCEDE III-atmospheric excitation by electron deposition, Eos Trans. $A G U, 71$, 1500

Paxton, L. J., and C.-I. Meng (1999), Auroral imaging and space-based optical remote sensing, Johns Hopkins APL Tech. Dig., 20, 556.

Pelaez, J., E. C. Lorenzini, O. López-Rebollal, and M. Ruiz (2000), A new kind of dynamic instability in electrodynamic tethers, J. Astronaut. Sci., $48,449$.

Rees, M. H. (1989), Physics and Chemistry of the Upper Atmosphere, Cambridge Univ. Press, New York.

Sanmartin, J. R., and R. D. Estes (1999), The orbital-motion-limited regime of cylindrical Langmuir probes, Phys. Plasmas, 6, 395.

Sanmartin, J. R., M. Martinez-Sanchez, and E. Ahedo (1993), Bare wire anodes for electrodynamic tethers, J. Propul. Power, 9, 353.

Sasaki, S., et al. (1985), Ignition of beam plasma discharge in the electron beam experiment in space, Geophys. Res. Lett., 12, 647.

Sasaki, S., et al. (1987), Results from a series of tethered rocket experiments, J. Spacecr., 24, 444.

Solomon, S. C., P. B. Hays, and V. J. Abreu (1984), Tomographic inversion of satellite photometry, Appl. Opt., 23, 3409 
Solomon, S. C., P. B. Hays, and V. J. Abreu (1985), Tomographic inversion of satellite photometry, part 2, Appl. Opt., 24, 4134.

Strangeway, R. J. (1980), On the instability of a spatially confined electron beam in a magnetized plasma, J. Plasma Phys., 24, 193.

Wilhelm, K., W. Stüdeman, and W. Riedler (1984), Electron flux intensity distributions observed in response to particle beam emissions, Science, $225,186$.

Winckler, J. R. (1980), The application of artificial electron beams to magnetospheric research, Rev. Geophys., 18, 659 .

Winckler, J. R. (1992), Controlled experiments in the Earth's magnetosphere with artificial electron beams, Rev. Mod. Phys., 64, 859.

Winckler, J. R., J. E. Steffen, P. R. Malcolm, K. N. Erickson, Y. Abe, and R. L. Swanson (1984), Ion resonances and elf wave production by an electron beam injected into the ionosphere: ECHO 6, J. Geophys. Res., 89,7565 .
Winglee, R. M., and P. L. Pritchett (1988), Comparative study of cross-field and field-aligned electron beams in active experiments, J. Geophys. Res., 93,5823

M. Charro, J. Pelaez, J. R. Sanmartin, and I. Tinao, Departamento de Fisica, Universidad Politecnica de Madrid, Madrid 28040, Spain (juanr.sanmartin.upm.es)

S. Elaskar, Departamento de Aeronautica, Universidad Nacional de Cordoba, Cordoba 2000, Argentina.

A. Hilgers, European Space Research and Technology Centre, European Space Agency, 2200 AG Noordwijk, Netherlands.

M. Martinez-Sanchez, Department of Aeronautics and Astronautics, Massachusetts Institute of Technology, Cambridge, MA 02139, USA. 